\title{
Synthesis of Acyclothymidine Triphosphate and $\alpha-P$-Boranotriphosphosphate and Their Substrate Properties with Retroviral Reverse Transcriptase
}

\author{
Ping Li, Mikhail Dobrikov, Hongyan Liu, and Barbara Ramsay Shaw*
}

Department of Chemistry, Box 90346, Duke University, Durham, North Carolina 27708-0346

brshaw@chem.duke.edu

\section{Spectral data for compounds $5 a$ and $5 b$.}

NMR were obtained on a Varian spectrometer operating at $400 \mathrm{MHz}$ for ${ }^{1} \mathrm{H}$ NMR and $161.9 \mathrm{MHz}$ for ${ }^{31} \mathrm{P}$ NMR in $\mathrm{D}_{2} \mathrm{O}$. The chemical shift for ${ }^{31} \mathrm{P}$ NMR was reported in ppm relative to $85 \% \mathrm{H}_{3} \mathrm{PO}_{4}$ as an external reference.

Acyclothymidine triphosphate (acTTP) 5a: ${ }^{1} \mathrm{H}$ NMR $\left(\mathrm{D}_{2} \mathrm{O}\right) \delta 7.44(\mathrm{~s}, 1 \mathrm{H}, \mathrm{H}-6), 5.09$ (s, 2H, NCH$)_{2}$, $3.96\left(\mathrm{~m}, 2 \mathrm{H}, \mathrm{P}(\mathrm{O}) \mathrm{OCH}_{2}\right), 3.67$ (m, 2H, $\left.\mathrm{CH}_{2} \mathrm{O}\right), 1.73\left(\mathrm{~s}, 3 \mathrm{H}, \mathrm{CH}_{3}-5\right) ;{ }^{31} \mathrm{P}$ NMR $\left(\mathrm{D}_{2} \mathrm{O}\right) \delta-9.72(\mathrm{~d}, 1 \mathrm{P}, \gamma-\mathrm{P}$, $\left.J_{\beta \gamma}=13.11 \mathrm{~Hz}\right),-10.07$ (d, 1P, $\left.\alpha-\mathrm{P}, J_{\alpha \beta}=13.44 \mathrm{~Hz}\right),-21.98(\mathrm{~m}, 1 \mathrm{P}, \beta-\mathrm{P}) ; \mathrm{UV}\left(\mathrm{H}_{2} \mathrm{O}\right) \lambda_{\max } 265.2 \mathrm{~nm}$; FAB-MS m/z 438.9 [(M-H)']; HRMS found: $\mathrm{m} / \mathrm{z} 438.9702$, calcd for $\mathrm{C}_{8} \mathrm{H}_{14} \mathrm{~N}_{2} \mathrm{O}_{13} \mathrm{P}_{3}{ }^{-}: 438.9709$.

Figure 1. RP-HPLC profile for ammonium salt of acyclothymidine triphosphate (acTTP) 5a

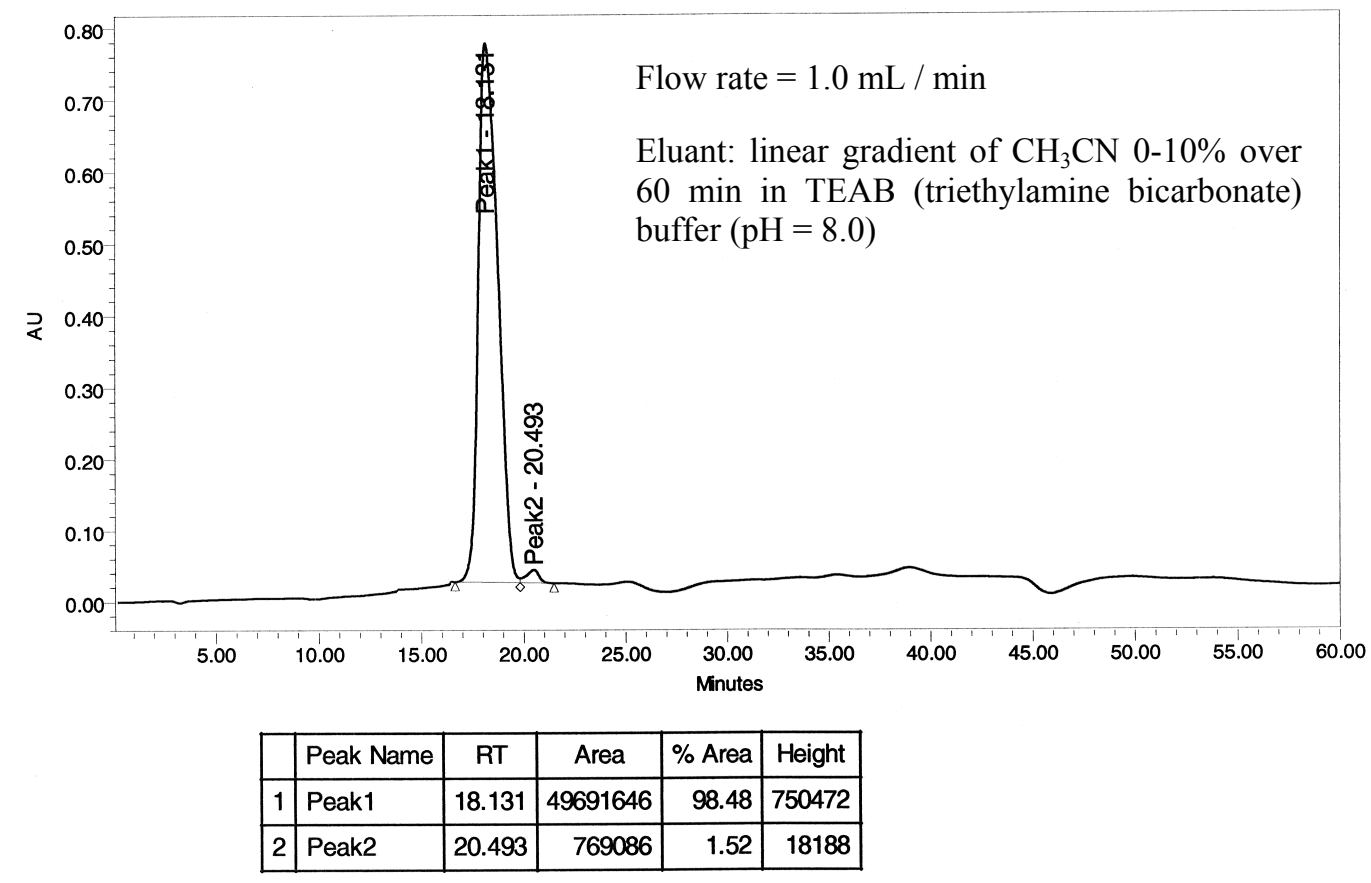


Acyclothymidine ( $\alpha$-P-borano)triphosphate (acTTP- $\alpha$ - $\left.B H_{3}\right) 5 \boldsymbol{b}:{ }^{1} \mathrm{H}$ NMR $\left(\mathrm{D}_{2} \mathrm{O}\right) \delta 7.42(\mathrm{~s}, 1 \mathrm{H}, \mathrm{H}-6)$, 5.06 (s, 2H, $\left.\mathrm{NCH}_{2}\right), 3.94$ (dt, $\left.J=10,4.8 \mathrm{~Hz}, 2 \mathrm{H}, \mathrm{P}(\mathrm{O}) \mathrm{OCH}_{2}\right), 3.67$ (t, $\left.J=4.8 \mathrm{~Hz}, 2 \mathrm{H}, \mathrm{CH}_{2} \mathrm{O}\right), 1.72$ (s, $\left.3 \mathrm{H}, \mathrm{CH}_{3}-5\right), 0.20$ (br, 3H, BH $)_{3}$; ${ }^{31} \mathrm{P}$ NMR $\left(\mathrm{D}_{2} \mathrm{O}\right) \delta 84.58$ (br, 1P, $\left.\alpha-\mathrm{P}\right),-6.13\left(\mathrm{~d}, 1 \mathrm{P}, \gamma-\mathrm{P}, J_{\beta \gamma}=24.45\right.$ Hz), -21.98 (t, 1P, $\beta-\mathrm{P}, J=21.69 \mathrm{~Hz}$ ); UV $\left(\mathrm{H}_{2} \mathrm{O}\right) \lambda_{\max } 265.2 \mathrm{~nm}$; FAB-MS m/z $437.0\left(\mathrm{M}^{-}\right) ; \mathrm{HRMS}$ found: $\mathrm{m} / \mathrm{z} 437.0101$ (for ${ }^{11} \mathrm{~B}$ ), calcd for $\mathrm{C}_{8} \mathrm{H}_{17} \mathrm{BN}_{2} \mathrm{O}_{12} \mathrm{P}_{3}^{-}: 437.0087$.

Figure 2. RP-HPLC profile for ammonium salt of acyclothymidine ( $\alpha$ - $P$-borano)triphosphate (acTTP- $\left.\alpha-\mathrm{BH}_{3}\right) \mathbf{5 b}$

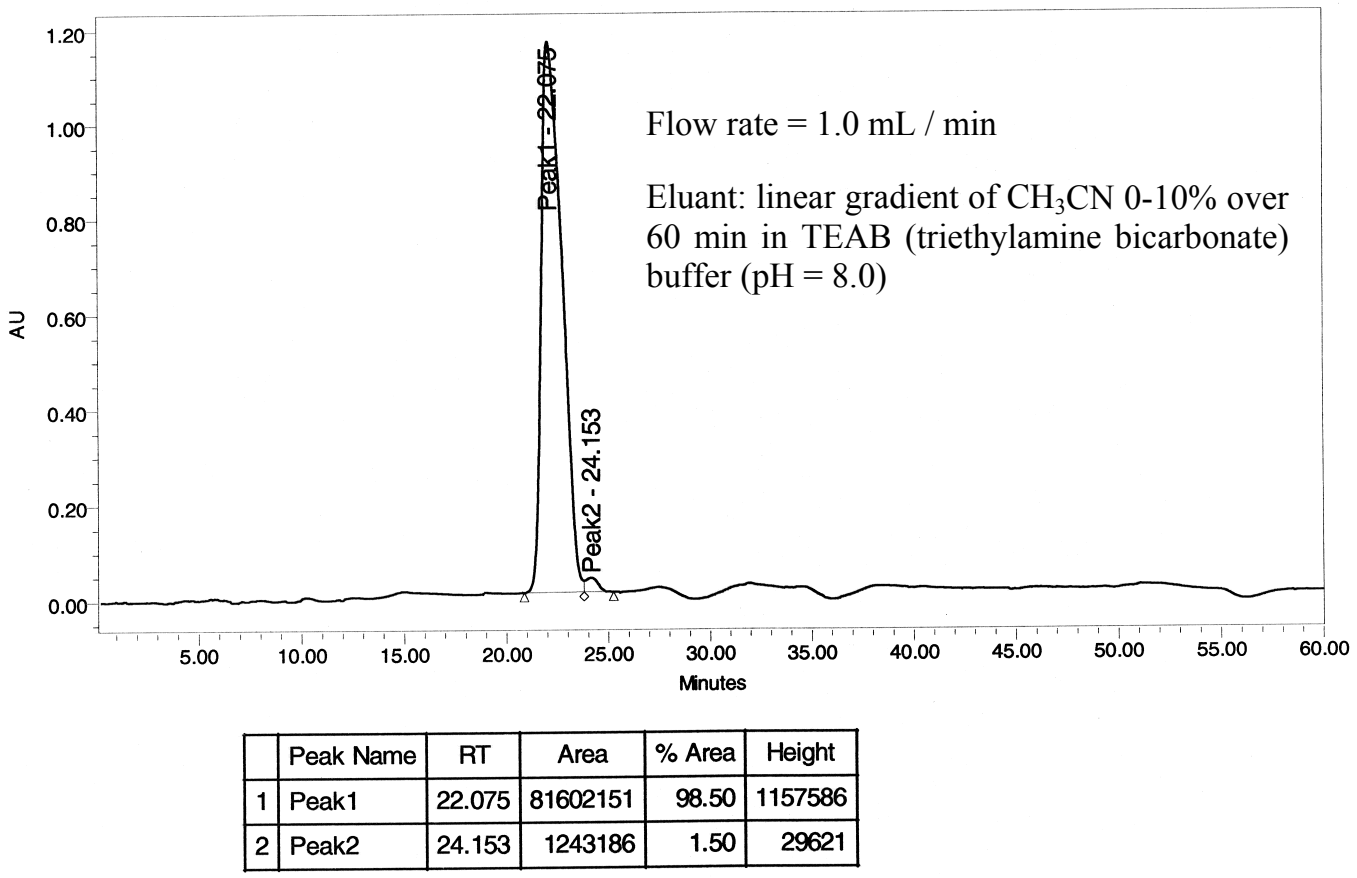

